\title{
Analysis, Mathematical Model and Selected Simulation Research of the GNSS Navigation Receiver Correlator
}

\author{
Lucjan Setlak ${ }^{*}$, Rafat Kowalik ${ }^{1^{*}}$
}

${ }^{1}$ Department of Avionics and Control Systems, Polish Air Force Academy, ul. Dywizjonu 303 nr 35, 08-521 Deblin

\begin{abstract}
The article presents a mathematical model describing the physical phenomena occurring in the GNSS radio receiver. The main subunit which focused attention was the system of GNSS receiver correlator. The process of tracking and acquiring navigation signals processed in a navigational radio receiver was analyzed in a special way. Bearing in mind that all kinds of noise and interference get through to the signals processed in the receiver, the article attempts to develop a mathematical model of the signal reaching the receiver with noise and an inappropriate signal. On the basis of the mathematical model developed, the courses of acquisition and tracking processes of SIS signals in the presence of noise and interference signal in the form of a narrow and broadband signal are presented. The final part the paper presents the results of computer simulations. Although the results presented in the paper refer to a specific GNSS receiver, they may differ depending on the types of radio navigation devices.
\end{abstract}

\section{Introduction}

The key component that is decisively influenced by the interfering signal in the Global Navigation Satellite System (GNSS) receiver is the correlator. In practical applications, it is responsible for the processes accompanying the determination of the correlation function between the code received together with the signal obtained from the satellite, and the one generated locally [1], [2], [3].

In view of the above, considering the importance of the correlator in satellite receivers, the authors in this paper have attempted to investigate how the said module reacts to the presence of undesired signals.

In addition, this system should also track received signals from the satellites that make up the space segment. The classic system that monitors the phase of the code in the navigation signal receivers is a DLL (Delay Lock Loop) system so-called the early-late gate.

The essence of the DLL operation is based on the correlation of the signal with three correspondingly delayed copies of the PRN (pseudo-random) code.

These copies are respectively early copies, corresponding to the code shifted to the left of the correlation maximum, prompt, defining the code in the correlation maximum (without phase change) and late, indicating the code shifted to the right from the correlation maximum [4], [5], [6].

Usually these shifts have values ranging from \pm 0.05 to \pm 0.5 chip. The block diagram of the analyzed GNSS receiver correlator is shown in the figure above (Fig. 1).

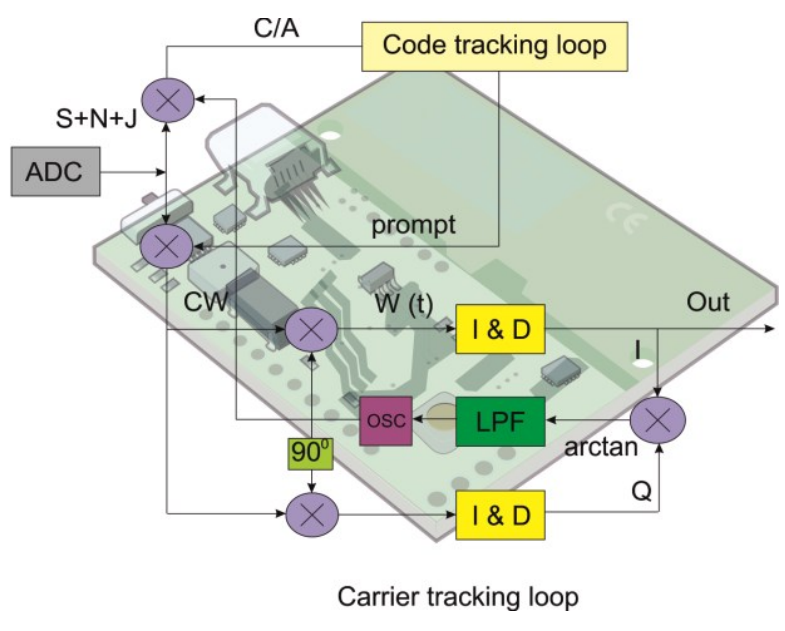

Fig. 1. Block diagram of the GNSS receiver correlator

Performing a detailed analysis of the correlator of the GNSS navigation receiver, including in particular its parameter in the form of the $\mathrm{C} / \mathrm{N}_{0}$ ratio, it is first necessary to consider the case of circumstances in which the $\mathrm{C} / \mathrm{N}_{0}$ ratio significantly exceeds the values accepted by the navigation receivers by the others.

In this case, the receiver is not able to correctly determine the correlation maximum of received codes. This function is carried out via the correlator of the navigation receiver on the basis of a comparison of received codes, the first code being the code received from the satellite together with the signal without taking into account SIS (Signal in Space) errors, while the second is the code generated locally by the receiver.

\footnotetext{
*Corresponding author: 1.setlak@wsosp.pl, r.kowalik@wsosp.pl
} 
It should be noted that the occurrence of an increased value of $\mathrm{C} / \mathrm{N}_{0}$ ratio in the receiver will cause that the procedure carried out in the DLL loop it is not able to properly determine the maximum correlation value, despite the use of specially designed codes for this purpose, generated locally in the navigation receiver (prompt, early and late). The point determined by the correlator in the form of the vertex of the correlation function always fluctuates close to the correct value.

This kind of situation results from two factors, namely: in the absence of synchronization between the code and the carrier wave generated by the satellite, and those generated locally and in the case of a difference between the carrier frequency and the frequency at which the interfering signal is transmitted.

In addition, it is also possible to move the previously generated code over time, however, this may result in reduction in value of the signal-to-noise ratio SNR (Signal-to-Noise Ratio). In the further part of the work, an analysis of the correlator of the GNSS navigation receiver was made in the scope of creating its mathematical description (model) [7], [8], [9].

\section{Analysis and Mathematical Model of the Navigation Receiver Correlator Implemented in Aviation}

\subsection{Tracking signals in the GNSS receiver in the presence of interfering signals}

In the analysis of the subject of the study, it was determined that it will be considered on the example of the GPS (Global Positioning System) receiver, among others due to its large spread and range of practical applications.

The SIS signal with a binary pseudorandom code (general access) C/A (Coarse Acquisition) received by the GPS receiver in the presence of an interfering signal can be described by the following formula [10], [11], [12].

$$
\begin{gathered}
S(t)=\sqrt{2 P_{S}} D(t) P_{N}(t) \cos \left(2 \pi f_{c} t+\varphi_{c}(t)\right) \\
+n(t)+j(t)
\end{gathered}
$$

where: $P_{s^{-}}$means the value of the signal power reaching the receiver, $D(t)$ - the navigational message transmitted at a rate of $50 \mathrm{bit} / \mathrm{s}, f_{c^{-}}$this is the carrier frequency for the GPS system, whereby it can take two values $f_{c}=$ $1575.42[\mathrm{MHz}]$ for the L1 band and $f_{c}=1227.6[\mathrm{MHz}]$ for the L2 band.

In turn using by $\varphi_{c^{-}}$the carrier wave angle is determined, $P_{N^{-}}$pseudorandom PRN sequence intended for transferring navigation data, $n(t)$ - it is the own noise of the receiver (represented in the analysis used white noise), in turn using $j(t)$ - an interfering signal was defined, which can be expressed, e.g. by a continuous wave described by the following equation:

$$
j(t)=\sqrt{2 J} \cos \left(2 \pi f_{i} t+\varphi_{i}(t)\right)
$$

where: $J$ - expresses the value of the power of the interfering signal emitted on the frequency $f_{i}$ and the angle $\varphi_{i}$.

In addition, it was assumed that the radio part of the front end navigation receiver receives both SIS beacons as well as an interfering signal, whereby the own noise of the receiver assumes a power equal to $\mathrm{N}_{0}$. In view of the above, for the purpose of determining the user's position, one can assess the $\mathrm{C} / \mathrm{N}_{0}$ relation by analyzing the operations performed by the correlator placed inside the navigation receiver and the tracking loop of the DLL code. Therefore, to determine the correlation function, the code received from a satellite should be compared with a code generated locally by the receiver [13], [14], [15].

This type of case can be described by the equation:

$$
P_{P N}(t)=P_{N}(t-\tau)
$$

where: $\tau$ - specifies the phase difference that is contained between the code received by the receiver and the one generated locally in the code necessary to determine the vertex of the autocorrelation function.

In turn, the carrier wave generated by the code tracking loop can be described by the following relationship:

$$
f_{\text {CARRIER }}(t)=\cos \left(2 \pi \widehat{f}_{c} t+\widehat{\varphi}_{c}\right)
$$

where: $\widehat{f}_{c}$ and $\widehat{\varphi}_{c^{-}}$- they are estimated values of the carrier frequency and the carrier wave angle generated locally by the navigation receiver.

In most cases, the wave is synchronized with a carrier wave received along with the SIS signal. The simulations carried out in the further part of the paper assume that the carrier waves are synchronized with each other. In addition, by performing the operation of multiplication of the signal with the reference signal with the carrier wave, in the procedure of determining the correlation function, in the integration operation the bit duration of the navigation message $T_{d}$, which is $1[\mathrm{~ms}]$ [16], [17].

For the needs of simpler calculations, the useful signal $S(t)$ considered before the integration operation is shown in complex form:

$$
S(t)=I_{S}(t)+j Q_{S}(t)
$$

where: $I_{s}(t)$ and $Q_{s}(t)$ - are respectively components, inphase and quadrature.

In view of initial assumptions, before the integration operation, the following were obtained:

$$
S(t)=I_{S}(t)+j Q_{S}(t)
$$

In this case, the signal in the correlator $W_{s}$ before performing the integration operation can be presented as:

$$
W_{s}(t)=\sqrt{2 P_{S}} P_{N}(t) P_{N}(t-\tau) e^{j\left(2 \pi \Delta f_{c} t+\Delta \varphi_{c}\right)}
$$

where: by $\Delta f_{c}$ and $\Delta \varphi_{c^{-}}$carrier errors were determined, associated with frequency change and its phase. 
Assuming that the difference between the phase of the carrier wave received by the receiver and the wave of the interfering signal is small, one can accept the condition $\Delta \varphi_{c}=0$.

Therefore, assuming that $C_{i}$ are the Fourier series coefficients of the periodic function $P_{N}(t)$ this case can be expressed by the following formula:

$$
P_{N}(t)=\sum_{i=-\infty}^{\infty} C_{i} e^{j 2 \pi \frac{i}{T_{c}} t}
$$

where: $T_{c^{-}}$is the code period, therefore the equation (7) can be written in the following form [18].

$$
\begin{aligned}
& W_{s}(t)=\sqrt{2 P_{s}} P_{N}(t) P_{N}(t-\tau) e^{j\left(2 \pi \Delta f_{c} t\right)} \\
& =\sum_{l=-\infty}^{\infty} \sum_{i=-\infty}^{\infty} C_{i} C_{l} e^{j 2 \pi\left[\frac{(i+l)}{T_{c}}+\Delta f_{c}\right]} e^{-j 2 \pi \frac{i}{T_{c}} t}
\end{aligned}
$$

By defining the new parameter $\mathrm{m}=\mathrm{i}+1$ and $R_{m}(t)=$ $\sum_{l=-\infty}^{\infty} C_{m-l} C_{l} e^{-j 2 \pi \frac{\tau}{T_{c}}}$ the following was received:

$$
W_{s}(t)=\sqrt{2 P_{s}} \sum_{m=-\infty}^{\infty} R_{m}(\tau) e^{j 2 \pi\left[\frac{m}{T_{c}}+\Delta f_{c}\right] t}
$$

As shown in the next figure (Fig. 2), the signals should be integrated, taking into account the duration of the period of the emitted code $T_{d}$ together with the SIS signal, which can be presented in the following form:

$$
\begin{aligned}
& \frac{1}{T_{d}} \int_{-\frac{T_{d}}{2}}^{\frac{T_{d}}{2}} P_{N}(t) P_{N}(t-\tau) e^{j 2 \pi \Delta f_{c} t} d t \\
& =\frac{1}{T_{d}} \sum_{m=-\infty}^{\infty} R_{m}(\tau) \int_{-\frac{T_{d}}{2}}^{\frac{T_{d}}{2}} e^{j 2 \pi\left[\frac{m}{T_{c}}+\Delta f_{c}\right] t} d t
\end{aligned}
$$

Solving the above equation was obtained [19], [20]:

$$
\begin{aligned}
& \int_{-\frac{T_{d}}{2}}^{\frac{T_{d}}{2}} e^{j 2 \pi\left[\frac{m}{T_{c}}+\Delta f_{c}\right] t} d t \\
& =\frac{1}{T_{d}} \frac{1}{2 \pi\left[\frac{m}{T_{c}}+\Delta f_{c}\right]} 2 \sin \left\{2 \pi\left[\frac{m}{T}+\Delta f_{c}\right] \frac{T_{d}}{2}\right\} \\
& =\operatorname{sinc}\left\{\left[\frac{m}{T_{c}}+\Delta f_{c}\right] T_{d}\right\}
\end{aligned}
$$

Substituting the obtained relation to formula (10) a new integral expression was obtained for the integral for the $W_{s}$ signal, which is as follows:

$$
\begin{gathered}
\int_{-\frac{T_{d}}{2}}^{\frac{T_{d}}{2}} W_{s}(t) d t=T_{d} \sqrt{2 P_{s}} \sum_{m=-\infty}^{\infty} R_{m}(\tau) \operatorname{sinc}\left\{\left[\frac{m}{T_{c}}\right.\right. \\
\left.\left.+\Delta f_{c}\right] T_{d}\right\}
\end{gathered}
$$

If you take into account the condition $\Delta f_{c} \ll \frac{1}{T_{c}}$ then

$$
\begin{aligned}
& \left|\int_{-\frac{T_{d}}{2}}^{\frac{T_{d}}{2}} \sqrt{2 P_{S}}(t) P_{N}(t-\tau) e^{j 2 \pi \Delta f_{c} t} d t\right| \\
& \cong \sqrt{2 P_{s}} T_{d} R_{0}(\tau) \operatorname{sinc}\left(\Delta f_{c} T_{d}\right)
\end{aligned}
$$

where:

$$
\begin{gathered}
\sum_{i=-\infty}^{\infty} C_{i} C_{l} e^{j 2 \pi\left[\frac{(i+l)}{T_{C}}+\Delta f_{c}\right]} e^{-j 2 \pi \frac{i}{T_{c}} t} \\
R_{0}(\tau)=\sum_{l=-\infty}^{\infty} C_{i} C_{l} e^{-j 2 \pi \frac{i}{T_{C}} \tau}
\end{gathered}
$$

In the next stage of the analysis, the signals received at the output of the correlator were analyzed in the presence of an interfering signal. In this case, the interfering signal is multiplied by the carrier wave, followed by its dissipation.

In turn, in the second phase, the same operation occurs, but with the difference that the received signal is again subjected to a multiplication operation, this time by the code generated locally by the receiver.

The resulting signal can be written as:

$$
\begin{aligned}
& W_{I}(t)=I(t) P_{N}(t-\tau) e^{j\left(2 \pi f_{c} t\right)} \\
& =\sum_{l=-\infty}^{\infty} \sum_{-\infty}^{\infty} J_{n} C_{l} e^{j 2 \pi\left[\frac{(n+l)}{T_{c}}+\Delta f_{i}\right] t} e^{-j 2 \pi \frac{l}{T_{c}} t}
\end{aligned}
$$

In the above equation (16) it was assumed that the frequency at which the interfering signal is emitted is marked as $f_{i}$, and the number of signals transmitted by $n$.

In contrast to the analogous activities carried out to determine the signal at the correlator output in the absence of an interfering signal, if it is present, the Doppler frequency $\Delta f i$, must be taken into account, which is received along with the L1 signal of the GPS system [21], [22].

This situation can be represented by the following relationships: $\widehat{f}_{c}-f_{i}=\Delta f_{i}+\frac{n}{T_{c}}$ and $\Delta f_{i}=f_{i}-\widehat{f}_{c}$.

In turn, after substituting for equation (16) and integration, it was obtained: 


$$
\begin{array}{r}
\int_{-\frac{T_{d}}{2}}^{\frac{T_{d}}{2}} W_{I}(t) d t=\sum_{l=-\infty}^{\infty} J_{n} C_{l} \sin c\left\{\left[\frac{n+l}{T_{c}}\right.\right. \\
\left.\left.+\Delta f_{i}\right] T_{d}\right\} e^{-j 2 \pi \frac{l}{T_{c}} t}
\end{array}
$$

Being aware that the frequency $\Delta f_{i}$ is significantly smaller than $\frac{1}{2 T_{c}}$, so for $n \neq-l$ the value of the function sinc is equal to zero.

$$
\operatorname{sinc}\left\{\left[\frac{n+l}{T_{c}}+\Delta f_{i}\right] T_{d}\right\} \cong 0
$$

Otherwise:

$$
\operatorname{sinc}\left\{\left[\frac{n+l}{T_{c}}+\Delta f_{i}\right] T_{d}\right\} \equiv \operatorname{sinc}\left(\Delta f_{i} T_{d}\right)
$$

Based on the above it was obtained:

$$
\int_{-\frac{T_{d}}{2}}^{\frac{T_{d}}{2}} W_{I}(t) d t=T_{d} J_{n} C_{l} \operatorname{sinc}\left(\Delta f T_{d}\right) e^{-j 2 \pi \frac{l}{T_{c}} t}
$$

Thus, according to the dependence (20) on the ratio $C / N_{0}$ in the presence of an interfering signal, it can be expressed as [23]:

$$
\frac{C}{N_{0}}=\frac{P_{s} G_{s}}{G_{n} N_{0}+J}
$$

where: $P_{s^{-}}$is the power value of the SIS signal received by the navigation receiver, $G_{s^{-}}$processing gain for the pseudorandom code scattering signal, $N_{0^{-}}$spectral noise power density, $G_{n^{-}}$noise processing gain and $J$ interference signal power.

Inserting equations (10) and (20) into the expression (21), an expression for the efficiency of signal-to-noise ratio SNR $\left(\mathrm{C} / \mathrm{N}_{0}\right)$ was obtained for the navigation receiver used on the modern aircraft [24], [25], [26].

$$
\frac{C}{N_{0}}=\frac{\left(\sqrt{2 P_{s}} T_{d} R_{0}(\tau) \operatorname{sinc}\left(\Delta f_{i} T_{d}\right)\right)^{2}}{G_{n} N_{0}+\left(J_{n} C_{l} \operatorname{sinc}\left(\Delta f T_{d}\right)\right)^{2}}
$$

\subsection{Determining the characteristics of erroneous bit reception for BOC modulation in the presence of a narrowband interfering signal on the GNSS receiver}

The process of determining the anti-interference characteristic for the BOC signal (Bit Processing of Composite), which is actively affected by the narrowband interfering signal, proceeds analogically as in the case of the broadband signal.

However, account should be taken of the fact that the narrowband interference signal band is several times narrower than in the case of a broadband signal band.
The bandwidth of the narrowband signal can be expressed by $\mathrm{W}_{\rho}=\rho \mathrm{W}$ the information transmission rate equal to $R_{b}$. In turn, the segment of the narrowband interfering signal is defined by the parameter $\rho$.

The spectral power density of the interfering signal can be determined from the equation $N_{0 J}^{\rho}=\frac{J}{\rho \mathrm{W}}=\frac{N_{0 J}}{\rho}$.

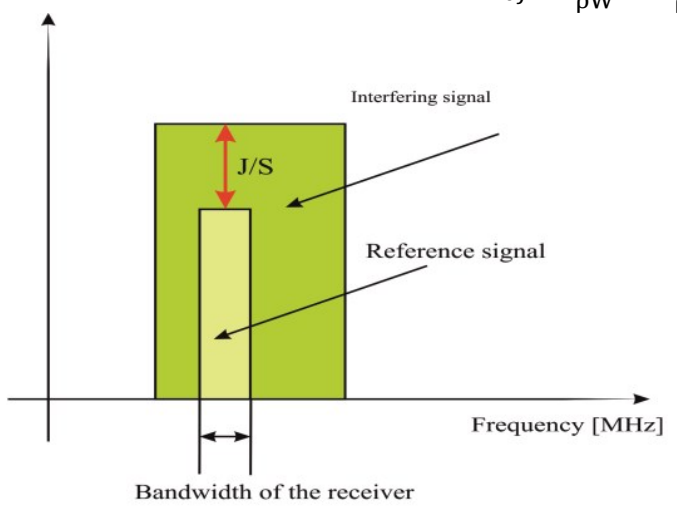

Fig. 2. Interpretation of the $\mathrm{J} / \mathrm{S}$ ratio in the range of the correct signal received by the receiver model

The useful power of the correct signal received by the receiver model shown in the figure above (Fig. 2) is $P$, and the interference signal power is $J$, while the duration of a single chip for the $P R N$ scattering signal used in BOC modulation is $T_{c}$. As a result, the signal received by the receiver, located in the vicinity of the device that emits a narrowband interfering signal, can be written in the following form:

$$
\begin{gathered}
y(t)=\sqrt{2 P} \cos \left[\omega_{0} t+\theta_{d}(t)+\theta_{P N}(t)+\theta\right] \\
+n(t)+n_{J}(t)
\end{gathered}
$$

However, the reference signal generated locally by the receiver can be written as:

$$
r(t)=2 \cos \left[\left(\omega_{0}-\omega_{I F}\right) t+\theta_{P N}(t)\right]
$$

As a result of the signal synchronization operation generated locally with the signal received by the receiver, the following were obtained:

$$
\begin{aligned}
u(t)=\sqrt{2 P} \cos [ & \left.\omega_{I F} t+\theta_{d}(t)+\theta\right] \\
& +2(n(t) \\
& \left.+n_{J}(t)\right) \cos \left[\left(\omega_{0}-\omega_{I F}\right) t\right. \\
& \left.+\theta_{P N}(t)\right]+O\left[\left(2 \omega_{0}-\omega_{I F}\right)\right]
\end{aligned}
$$

In turn, as a result of passing the $u(t)$ signal through a band-pass filter a signal was obtained:

$$
\begin{aligned}
v(t)=\sqrt{2 P} \cos [ & \left.\omega_{I F} t+\theta_{d}(t)+\theta\right] \\
& +2(n(t) \\
& \left.+n_{J}(t)\right) \cos \left[\left(\omega_{0}-\omega_{I F}\right) t\right. \\
& \left.+\theta_{P N}(t)\right]
\end{aligned}
$$


The expression (26) above also contains the intermediate pulsation component $\omega_{I F}$. The signal after passing through the filter was divided into three components. The first part in formula (26) is the signal obtained by the focusing operation of the correct signal, the second component was shaped in the course of the narrowband interference signal concentration. The last third part was created by focusing the spectrum of own noise generated by the receiver. On the basis of the above analysis, in the discussed situation the spectral density of the own noise produced by the receiver can be calculated from the following relationship:

$$
S\left(f_{I F}\right)=\alpha \frac{N_{0}}{2}
$$

As it is already known, the coefficient $\alpha$ oscillates close to one, hence the value of the product $W T_{c}$ is three or four times greater. Taking into account the discussed properties, one can determine the spectral power density of a narrowband interfering signal after a cluster operation, which will be the same as it was in the broadband signal. Hence, as a result of the spectral focusing operation, the spectral power density of the reference signal can be described by the following relationship.

$$
\begin{aligned}
S_{r}(f)=T_{c} \operatorname{sinc}^{2}[ & \left.\left(f-f_{0}+f_{I F}\right) T_{c}\right] \\
& +T_{c} \operatorname{sinc}^{2}\left[\left(f+f_{0}-f_{I F}\right) T_{c}\right]
\end{aligned}
$$

In a situation in which the interfering signal and obtained sequence obtained as a result of spectral cluster operations are uncorrelated with each other, the spectral power density of the interfering signal can be written as:

$$
S_{J P N}(f)=\int_{-\infty}^{\infty} S_{r}\left(f-f^{\prime}\right) S_{J}\left(f^{\prime}\right) d f^{\prime}
$$

In summary, when the bandwidth of the narrowband interfering signal is $\rho W[\mathrm{~Hz}]$, then the spectral power density of the undesired signal can be determined from the equation $\frac{N_{0 J}^{\rho}}{2}=\frac{J}{\rho W} \mathrm{~W} / \mathrm{Hz}$, where: $J$ - is the power of the interfering signal, and $f_{J}$ - the center frequency of the undesired signal emission. In this way, the spectral power density of the interfering signal whose center frequency is $-f_{J}$ or $f_{J}$ is described by the following relationship:

$$
\begin{aligned}
& S_{J}(f)=\frac{N_{0 J}^{\rho}}{2} d l a\left|f+f_{J}\right| \leq \frac{\rho W}{2} \\
& S_{J}(f)=\frac{N_{0 J}^{\rho}}{2} \text { dla }\left|f-f_{J}\right| \leq \frac{\rho W}{2}
\end{aligned}
$$

Using the earlier derivations and formulas (27), (28) and (30), the expression (29) can also be defined as:

$$
\begin{aligned}
S_{J P N}(f)=\frac{N_{0 J}^{\rho} T_{c}}{2} & \int_{f_{J}-\frac{\rho W}{2}}^{f_{J}^{+\frac{\rho W}{2}}}\left\{\operatorname { s i n c } ^ { 2 } \left[\left(f-f_{0}+f_{I F}\right.\right.\right. \\
& \left.\left.-f^{\prime}\right) T_{c}\right] \\
& +\sin c^{2}\left[\left(f+f_{0}-f_{I F}\right.\right. \\
& \left.\left.\left.-f^{\prime}\right) T_{c}\right]\right\} d f^{\prime} \\
& +\frac{N_{0 J}^{\rho} T_{c}}{2} \int_{-f_{J}^{+\frac{\rho W}{2}}}\left\{\operatorname{sinc}^{2}[(f\right. \\
& -f_{0}+f_{I F}-\frac{\rho W}{2} \\
& +\operatorname{sinc}^{2}\left[\left(f+f_{c}-f_{I F}\right]\right. \\
& \left.\left.\left.-f^{\prime}\right) T_{c}\right]\right\} d f^{\prime}
\end{aligned}
$$

Assuming that the frequencies $f_{0}$ and $f_{J}$ are not directly in the range of the interference signal band $\rho W$ [Hz], and the condition $f_{0} \gg 2 f_{I F}$ is met, then in this case for $f=+f_{I F}$ the second member in the first integral is equal to one.

In turn, for $f=-f_{I F}$ the third integral in the second integral is reduced, which results in the dissipation of the spectral power density for the interfering signal into two areas located at the center frequencies $f_{I F}$ and $-f_{I F}$.

Hence the spectral density of the interfering signal power for frequency $f_{I F}$ will take the following form:

$$
S_{J P N}\left(f_{I F}\right)=\frac{N_{0 J}^{\rho} T_{c}}{2} \int_{\substack{\left.f_{J}-\frac{\rho W}{2} \\-f^{\prime}\right) T_{c}}}^{f^{+\frac{\rho W}{2}}} \operatorname{sinc}^{2}\left[\left(f_{0}\right.\right.
$$

and for frequency $-f_{I F}$

$$
\begin{aligned}
S_{J P N}\left(-f_{I F}\right)=\frac{N_{0 J}^{\rho} T_{c}}{2} & \int_{-f_{J}-\frac{\rho W}{2}}^{-f_{J}+\frac{\rho W}{2}} \operatorname{sinc}^{2}\left[\left(-f_{0}\right.\right. \\
& \left.\left.-f^{\prime}\right) T_{c}\right] d f^{\prime}
\end{aligned}
$$

Based on the above, it can be observed that the above two integrals are very similar, so the spectral power density of the noise should be written as:

$$
\begin{aligned}
S_{J P N}\left(f_{I F}\right)=S_{J P N} & \left(-f_{I F}\right) \\
& =\frac{N_{0 J}^{\rho} T_{c}}{2} \int_{f_{J}-\frac{\rho W}{2}}^{f_{J}+\frac{\rho W}{2}} \operatorname{sinc}^{2}\left[\left(f_{0}\right.\right. \\
& \left.\left.-f^{\prime}\right) T_{c}\right] d f^{\prime}
\end{aligned}
$$


Expression (34) is true in the case when the bandwidth of the interfering signal is large enough to cause errors in the proper reception of the correct signal. In addition, in the band $\rho W$ affect the frequencies $f_{J}$ and $f_{0}$ of the same values, so the condition $f_{0} \gg 2 f_{I F}$ is met.

Considering the above, it can be seen that there are two possibilities to use the narrowband interference signal band.

In the first case, the band of the interfering signal $\rho W$ is quite narrow compared to the bit rate transmitted $\frac{1}{T_{c}}$.

In such a situation, neighboring usable signals may also enter the interference band.

Therefore, the spectral noise power density $S_{J P N}\left(f_{I F}\right)$ is expressed as follows:

$$
\begin{array}{r}
S_{J P N}\left(f_{I F}\right)=\frac{N_{0 J}^{\rho} T_{c} \rho W}{2}\left[\operatorname{sinc}^{2}\left(f_{J}-f^{\prime}\right) T_{c}\right]^{2} \\
=\frac{J T_{c}}{2}\left[\operatorname{sinc}^{2}\left(f_{J}-f^{\prime}\right) T_{c}\right]^{2}
\end{array}
$$

Considering the fact that the interfering signal is the most effective when the operating frequency of the interfering signal is the same as the frequency of the correct signal, then in such a situation the spectral power density of noise is equal to:

$$
S_{J P N}\left(f_{I F}\right)=\frac{J T_{c}}{2}
$$

Hence, setting the same frequency of transmission (working) of the interfering signal as the proper signal results in a significant improvement of its effectiveness.

Otherwise, the use of a narrowband interfering signal in the circumstances is unfounded.

As a consequence, further polemics over the analysis of the interfering signal with such a narrow band is pointless.

At this point, more attention should be paid to the second condition in which the parameter characterizing the interfering signal band $\rho=1$.

Consequently, in expression (35) a new variable $z=$ $\pi T_{c}\left(f_{0}-f^{\prime}\right)$, was introduced, assuming that $f_{0}=f_{J}$.

Therefore, the spectral signal power density is expressed as:

$$
S_{J P N}\left(f_{I F}\right)=\frac{J T_{c}}{2 W} \int_{-\pi T_{c}\left(\frac{W}{2}\right)}^{\pi T_{c}\left(\frac{W}{2}\right)} \frac{\sin ^{2}(z)}{z^{2} \pi T_{c}} d z
$$

The calculation of the above integral and and its definition is very simple, so it was obtained:

$$
S_{J P N}\left(f_{I F}\right) \cong \frac{J}{2 W}
$$

Now that the $W$ band is equal $W=\frac{2}{T_{c}}$, the following is obtained:

$$
S_{J P N}\left(f_{I F}\right) \cong \frac{J T_{c}}{2 W}=\frac{J}{4 R_{c}}
$$

where: by $R_{c}$ - the speed of the dissipative line is determined $R_{c}=\frac{1}{T_{c}}$.

By comparing the expressions determining the spectral noise density (34) and (35) it can be observed that in a situation where the frequency of the interfering signal is equal to the carrier frequency $f_{0}$.

The narrowband interfering signal is more effective than the broadband signal with identical characteristic parameters (by almost $3 \mathrm{~dB}$ ). $\frac{2}{T_{C}}$.

This statement is correct on the assumption that $W=$

Therefore, to make it easier to determine the bit error rate for a BOC modulated signal, which is affected by the intentional interfering signal, it seems advantageous to assume that the narrowband interfering signal is represented by Gaussian noise.

In this case, the bit error rate BER (Bit Error Rate) can be determined from the following dependence:

$$
P_{b}=Q\left[\sqrt{\frac{E_{b}}{\frac{N_{0}}{2}+S_{J P N}\left(f_{I F}\right)}}\right]
$$

where: the spectral power density of the noise is $\frac{N_{0}}{2}$, in turn the spectral power density of the interfering signal is equal $S_{J P N}\left(f_{I F}\right)$.

According to the theory of signals, it was noticed that in this case there are two statistically independent stochastic processes accompanied by Gaussian noise.

In conditions where the narrowband interfering signal is transmitted at the same frequency as the actual signal, the bit error rate can be determined from the following equation:

$$
\begin{aligned}
P_{b}=Q\left[\sqrt{\frac{2 E_{b}}{N_{0}+J T_{c}}}\right] \\
=Q\left[\sqrt{\left.\frac{2}{\left(\frac{E_{b}}{N_{0}}\right)^{-1}+\left(\frac{P R_{c}}{J R_{b}}\right)^{-1}}\right]}\right. \\
=Q\left[\sqrt{\frac{2}{\left(\frac{E_{b}}{N_{0}}\right)^{-1}+\left(\frac{P}{J} G_{p}\right)^{-1}}}\right]
\end{aligned}
$$

It should be noted that the expression (41) is compatible with reality only when the system operates on the basis of the spectral scattering technique using the Direct Sequence DS (Direct Sequence) method, and the processing profit $G_{p}$ is equal to:

$$
G_{p}=\frac{R_{c}}{R_{b}}
$$


Based on the above considerations and analyzes, it can be concluded that the higher the processing profit, the lower the bit error rate and vice versa.

The next figure (Fig. 3) presents examples of bit rate results errors depending on the function $\left(P R_{c} / J R_{b}\right)$ for the modulated BOC signal in the presence of a narrowband interfering signal.

\section{Selected Simulation of the GNSS Navigation Receiver Correlator}

The operation of a typical GNSS receiver system is based on two processes, namely: the signal acquisition process, which includes the initial synchronization of the receiver radio reception circuitry with the signal and on the signal tracking process, whose essence is the continuous synchronization of reception systems of the receiver with a signal received from the satellite.

The acquisition of radio signals received from the satellite, due to the use by satellites of all transmitters of the same frequency is more complex than in typical radio receivers, as a result of which after the receiver is turned on, the phase of acquisition of GNSS signals begins.

During this process key activities are carried out, including determination of the expected configuration of the satellites, pre-setting the frequency of the generator in the receiver phase loops, turning on phase and code loop adjustment, and bit synchronization of signals and determining the position and corrections of the receiver clock time.

When making selected simulations of the GNSS navigation receiver in the scope of the acquisition and tracking process, it should be noted that in the case of the process of signal acquisition and processing, a number of decisions are made that have a significant impact on determining the position of the user.

Hence, any interferences (disturbances) of this process may be the cause of errors in the correct synchronization of angles, received from the satellite, including errors generated locally by the receiver. In the process of signal acquisition and processing, a number of decisions are made that have a significant impact on the determination of the user's position.

Therefore, disturbing this process may also result in errors in the correct synchronization of codes received from the satellite, including those generated locally by the receiver.

All simulation tests were carried out based on the author's interfering signal, which was transmitted on the center frequency of each tested signal with a power of $125 \mathrm{dBm}$.

Obtained results from the conducted simulation tests are presented on the above figures (Figures 3-4).

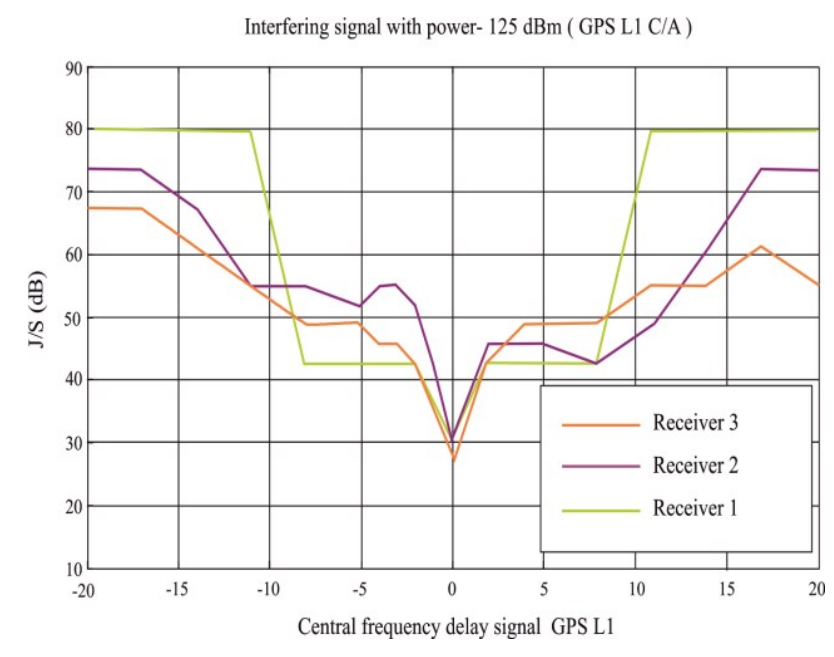

Fig. 3. Implementation of the L1 signal acquisition process in the presence of an interfering signal with power- $125 \mathrm{dBm}$ (GPS L1 (C/A)

When analyzing the above waveforms (Fig. 3), it should be explained at the outset that the point " 0 " means the value of the center frequency of the GNSS system signal under investigation, and the interfering signal continuously affected precisely this point during the tests. Moreover, the acquisition process in this case was clearly disturbed, while the pseudo-random sequence undergoes significant deformation.

There was a difference between individual values of the $\mathrm{J} / \mathrm{S}$ ratio level obtained for the receiving the same codes results from the fact that the tested receivers rated (characterized) various hardware parameters. It is known that the acquisition of signals in a GNSS receiver is optimal if the level of $\mathrm{C} / \mathrm{N}_{0}$ ratio is the lowest.

There are, however, receivers that, after exceeding the set value, the signal acquisition procedure misinterprets them. The quantity of $\mathrm{C} / \mathrm{N}_{0}$ for the tested receivers is as follows: No. 1: $19(\mathrm{~dB}-\mathrm{Hz})$, No. 2: 20 $(\mathrm{dB}-\mathrm{Hz})$ and for the receiver No. 3: 24 (dB-Hz).

The same procedure was adopted in the next test, only with the difference that in this case the tested receivers only received signals transmitted on the L1 frequency, only the value of the interfering signal power was changed to a value of $110 \mathrm{dBm}$.

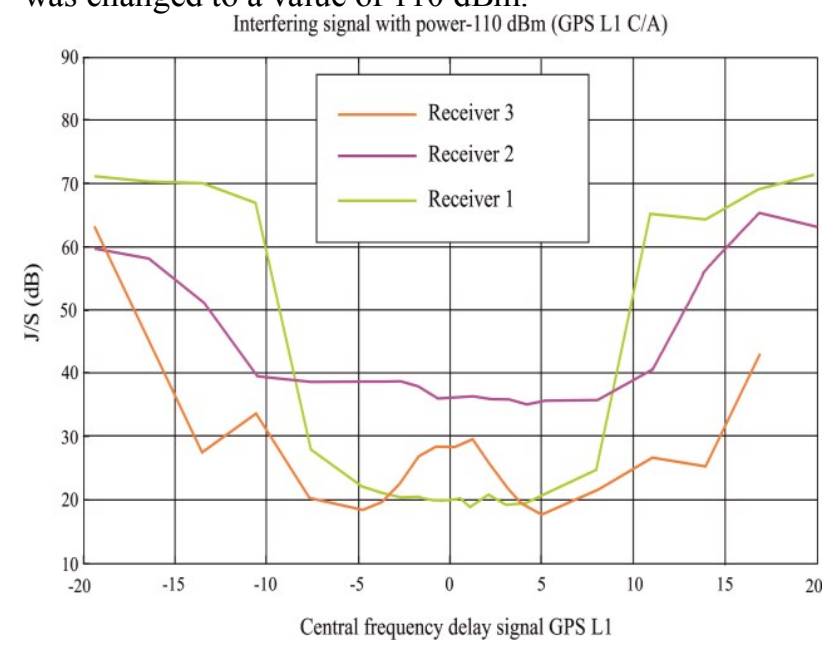

Fig. 4. Implementation of the $\mathrm{L} 1$ signal acquisition process in the presence of an interfering signal with power- $110 \mathrm{dBm}$ (GPS L1 (C/A) 
As can be seen from the above figure (Fig. 4), the procedure of signal acquisition is even more distorted than it was in the previous experiment. This is only due to the increase in the useful power of the interfering signal by less than $15 \mathrm{dBm}$. Subsequently, the level of the $\mathrm{J} / \mathrm{S}$ ratio at which the receiver misinterpreted the acquisition process, the signal also changed and focused on the values of $40 \mathrm{~dB}$ for receiver No. 1, $60 \mathrm{~dB}$ for receiver No. 2 and $63 \mathrm{~dB}$ for receiver No. 3, respectively. In the next simulation test, an acquisition analysis was performed for the L1 signal of the GPS system and L1 system of the Galileo system. Interfering signal with power $-125 \mathrm{dBm}$ (GPS, Galileo)

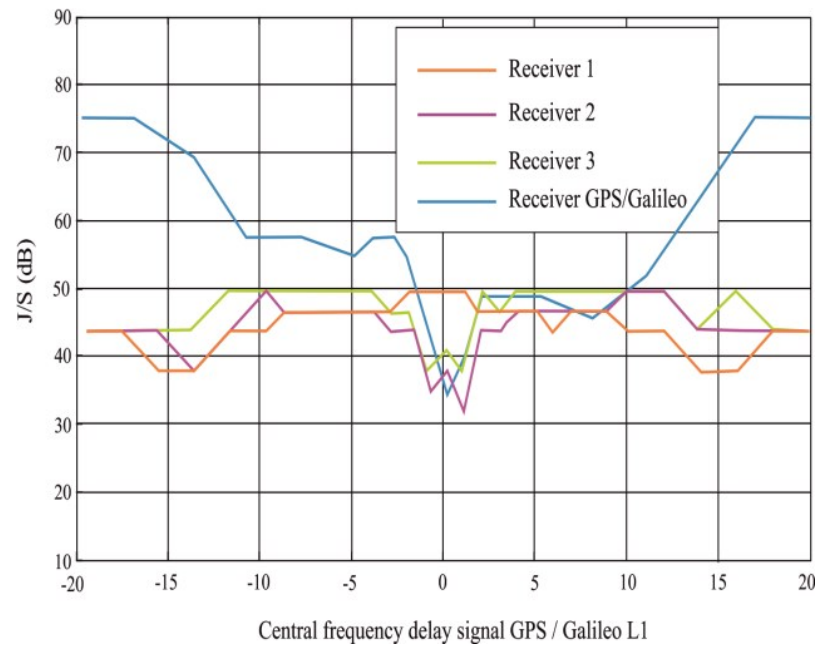

Fig. 5. Implementation of the L1 signal acquisition of the GPS and Galileo systems in the presence of an interfering signal with power- $125 \mathrm{dBm}$ (GPS, Galileo)

A combination of this kind will allow you to estimate how it reacts to signals interfering with the GPS/Galileo hybrid receiver. The power of the interfering signal was set at $-125 \mathrm{dBm}$ and was given at the center frequency of the L1 signal, and the test results obtained at this stage were depicted in the figure below (Fig. 5).

\subsection{Interfering with the process of tracking signals}

Analyzing the above graphs, it was noted that receivers receiving only signals from the Galileo system are more exposed to the interference signal. This is also due to the fact that they have a much wider $40 \mathrm{MHz}$ receive band than GPS receivers. Nevertheless, the arrangement of intertwined (mixed) codes in the Galileo signal undergoes minimal deformation, the codes coincided with the GPS system signal are much more disturbed. This fact is also reflected at the level of $\mathrm{J} / \mathrm{S}$ ratio, at which the tested receivers mistakenly acquire signals, and it is $75 \mathrm{~dB}$ for the L1 signal of the GPS system and $45 \mathrm{~dB}$ for the Galileo system. In addition, for the Galileo system receivers, an analysis for the individual code components contained in the main code is still to be carried out. This type of case was analyzed for the E6 and E5 signals, whereby they can be divided as follows: E6 signal into three E6A, E6B and E6C channels, and E5 signal for a code channel also called a pilot channel and a data channel. In all channels, a code signal will be transmitted, allowing to determine the distance of the satellite to the GNSS receiver, whereby only distance codes are transmitted in code channels. In turn, in the data channels, in addition to the distance codes, navigational messages are also transmitted. The proposed method of evaluating the process of acquisition of signals by the GNSS receiver in the case of activity of the interfering signal is based on the evaluation of the level of the J/S ratio. The results obtained at this stage of the simulation for the above case are presented in the following figures (Figures 6-7).

Interfering signal with power - $125 \mathrm{dBm}$ (Galileo E6)

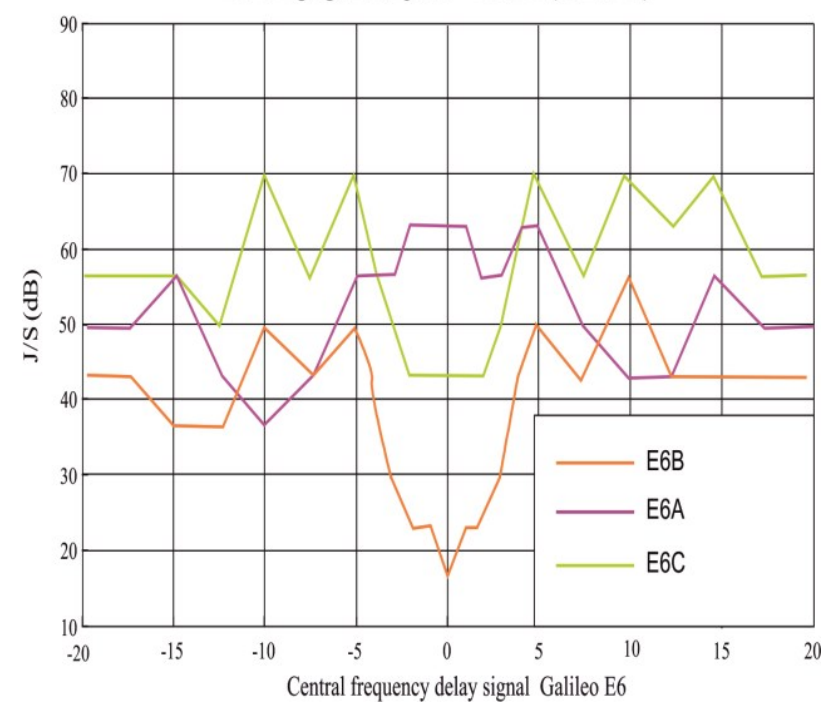

Fig. 6. Implementation of the Galileo E6 signal acquisition process in the presence of an interfering signal with power- 125 dBm (Galileo E6)

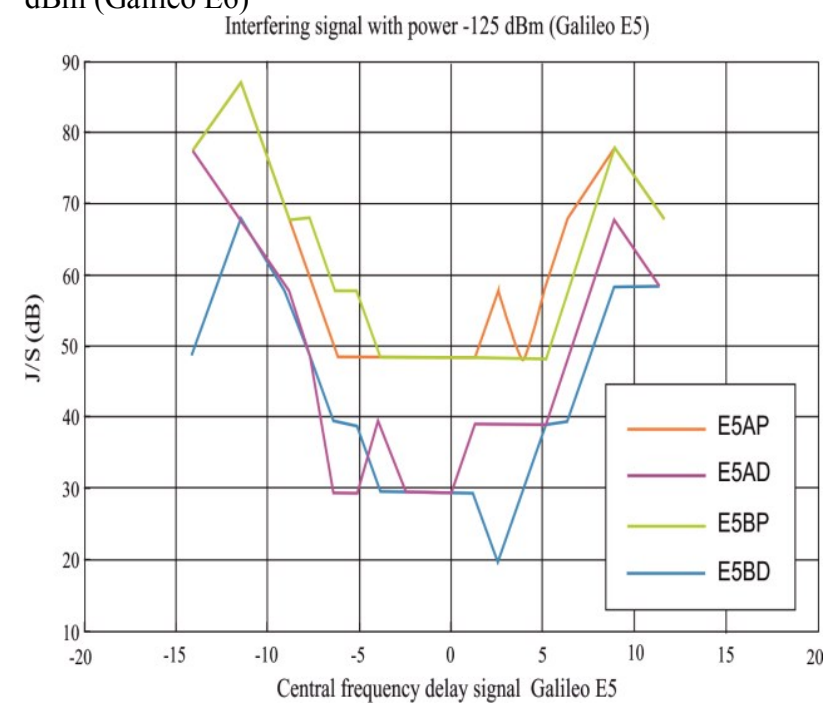

Fig. 7. Implementation of the Galileo E5 signal acquisition process in the presence of an interfering signal with power- 125 dBm (Galileo E5)

Based on the analysis of the above waveforms (Figures 6-7), it can be seen that the codes contained in Galileo signals are more resistant to interference signals. While in the case of the E6 signal all codes included in the main code have similar properties, the E5 signal component codes react quite differently. As can be seen, the E5A signal is not a reflection of the E5B signal. This can be seen, among others from the asymmetry created 
at the output of the filter or the fact that the receiver can not cope with the processing of both signals simultaneously. In sum, all the experiments carried out show that the signal developed causes disturbances in the acquisition process, however the receiver in which it is implemented (GPS or Galileo) is irrelevant.

\subsection{Stages of the signal acquisition process}

An analogous course of reasoning as for the process of signal acquisition was adopted in an experiment aimed at discerning discrepancies in the process of tracking codes by the GNSS receiver in the case where the tested receiver is exposed to the interference signal developed for the purposes of this article. For this purpose, the same satellite navigation receivers were used as in the previous experiment, however, in their deliberations, the authors limited themselves only to receivers operating on the L1 frequencies for the GPS system and E6 and E5 for the Galileo system [27], [28].

The area of simulation research covered key stages, including generating a interfering signal, turning on the GPS receiver and starting post-processing processes, launching the signal tracking loop and determining the autocorrelation function in the presence of an interfering signal. For this purpose, assumptions were made for the needs of the research that the GNSS receiver tested at a given moment would receive only the recommended signal (L1, E5 or E6).

Therefore, the results obtained will be more transparent and everyone will be able to assess them themselves, including people without specialist knowledge in this field. This was necessary primarily due to the long time of almanac synchronization of the receiver with the simulator, with the interfering signal being transmitted at $127 \mathrm{~dB}$ continuously. The results obtained from the conducted simulations and tests are presented in the figures below in the form of selected illustrations (Figures 8-13).

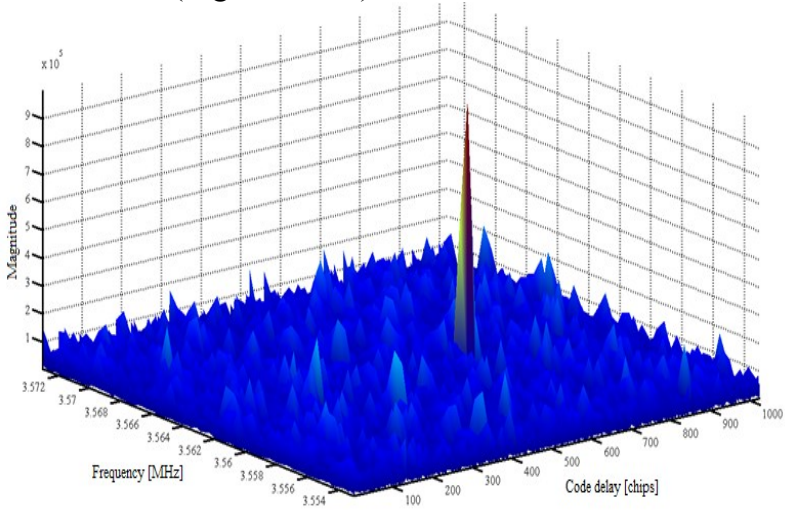

Fig. 8. Illustration of the code tracking process in the L1 signal of the GPS system and determination of the autocorrelation function in the event that the interfering signal is not functioning

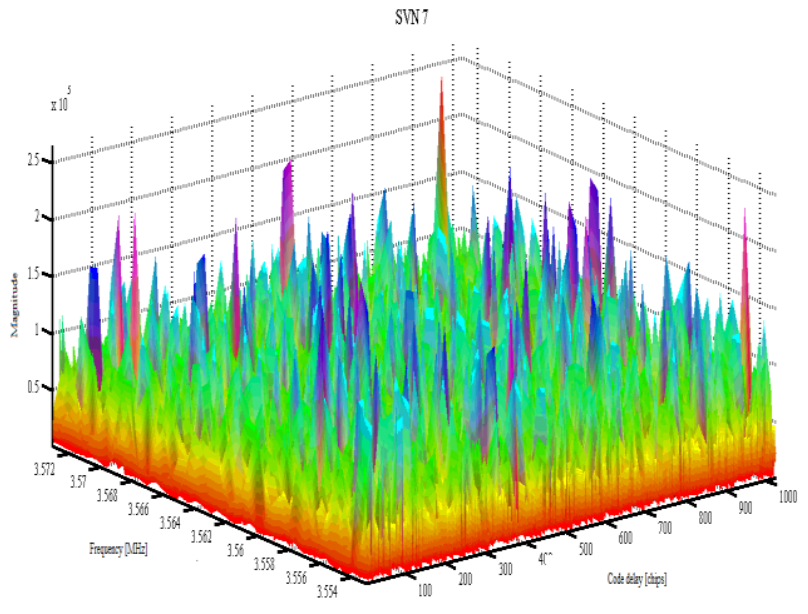

Fig. 9. Illustration of the tracking process of the SVN 7 code transmitted in the L1 signal of the GPS system and determining the autocorrelation function in the situation when the interfering signal is turned on

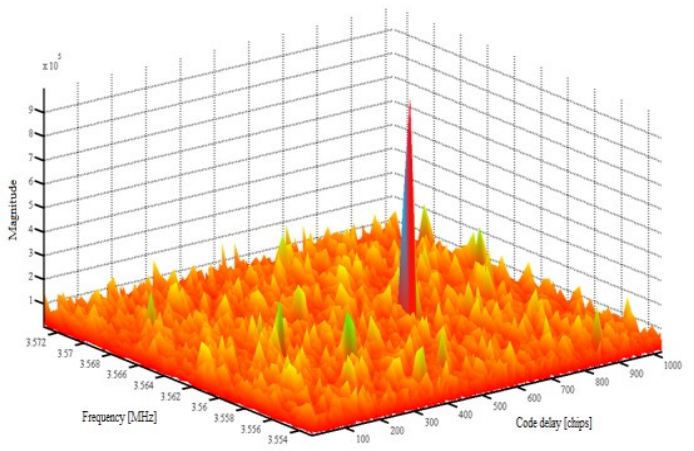

Fig. 10. Illustration of the code tracking process in the E5 signal of the Galileo system and determination of the autocorrelation function in the event that the interfering signal does not operate

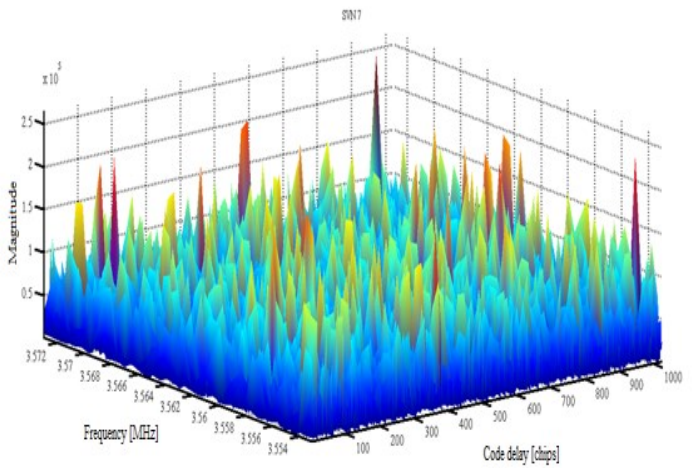

Fig. 11. Illustration of the code tracking process in the E5 signal of the Galileo system and determination of the autocorrelation function in the situation of the interfering signal activity 


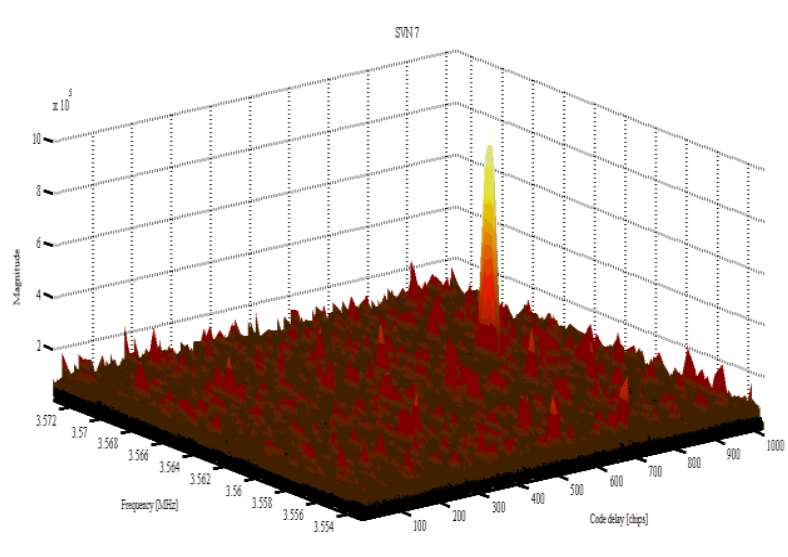

Fig. 12. Illustration of the code tracking process in the E6 signal of the Galileo system and determination of the autocorrelation function in the event that the interfering signal does not operate

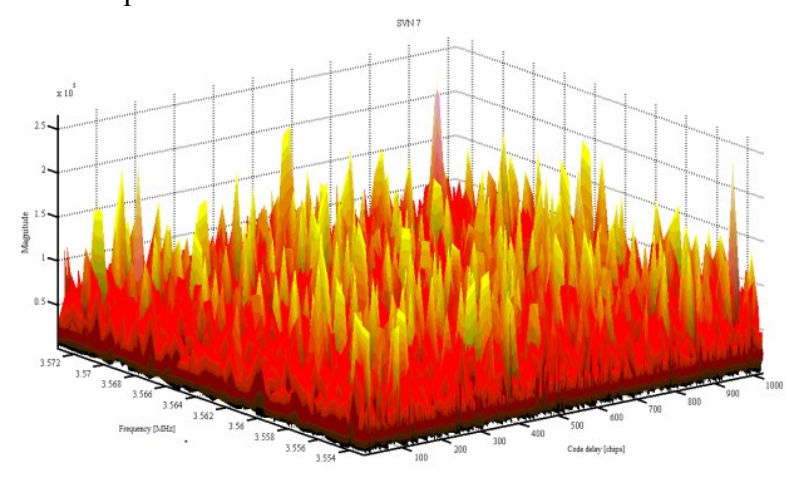

Fig. 13. Illustration of the code tracking process in the E6 signal of the Galileo system and determination of the autocorrelation function in the situation of the interfering signal activity

The obtained results confirm the effectiveness of the developed interfering signal, and the signal can be successfully used for disturbance, both GPS system signal and Galileo signals. Based on the presented illustrations, one can observe how the receiver can mistakenly determine the "beep" of the autocorrelation function, as two identical correlation "beeps" have been obtained as a result of preliminary code tracking. This kind of situation occurs because the structure of the interfering signal is similar to the structure of the signal.

\section{Conclusions}

The article presents the most important information that could be a guide for selecting the best correlator system in the satellite navigation receiver. The subjective choice of issues and the author's assessment of the essence of the presented parameters and functionalities of the process of acquisition and tracking of navigation signals by the GNSS receiver is only the preliminary stage of the research that was carried out.

The main parameter subjected to the tests through experiments and simulation tests was the impact of broadband interference signals on the work of the correlator system. It should be noted that the undesirable signals pose a potential serious threat to the security of devices and systems that use the information contained in signals broadcast by satellites of global navigation systems.

The implementation of such attacks can be particularly dangerous in areas such as: navigation (land, sea, air and space), energetics or telecommunications. Interference in the form of narrow and broadband signals is an intentional or unintentional signal that directly interferes with the signal in the frequency band of GNSS systems, while these systems have some internal mechanisms that significantly increase its resistance to interference. One of them is the choice of signal transmission based on the technique of spectrum scattering in broadband systems using DSSS (Direct Sequence Spread Spectrum) code sequences.

It is a process of modulation of the signal with direct data encryption by a pseudo-random sequence, to which one of the key advantages is, among others, resistance to interference. One of the elements of the GPS system modernization process is the inclusion of an additional signal frequency, which will be better adapted to counteract the interference due to the higher power [29], [30].

Currently, many systems for determining position, speed and time are based on the GPS system, which one of the most important tasks is protection of integrity, access, interferences detection and location of their sources, as well as counteracting these interferences. One of the conclusions resulting from the research presented in the article is, among others, the fact that, if the necessary activities (actions) are not taken to ensure adequate protection of navigation receivers, GNSS satellite navigation systems should not be used as the only sources of information about location, speed and time in the so-called critical applications.

In addition, among the factors that make it possible to distinguish between signals containing interference signals and real GNSS signals, the direction of signal arrival and its derivatives is the most unambiguous and the most difficult to "falsify".

Knowledge of exact phase delays of false signals enables not only detection of undesired signals in the correlator system, but also selective elimination of these signals from the entire signal received through the application of filtration using the null forming algorithm (null steering), which causes attenuation of signals reaching the antenna array from a particular direction.

\section{References}

1. Joseph L. Awange, "Environmental Monitoring using GNSS: Global Navigation Satellite Systems," Springer 2012

2. B. Hofmann-Wellenhof, H. Lichtenegger, E. Wasle, "GNSS - Global Navigation Satellite Systems: GPS, GLONASS, Galileo, and more, " Springer 2008

3. T. Pany, "Navigation Signal Processing for GNSS Software Receivers, " Artech House 2010 
4. O. Yigit, K. Yegin, "All-band GNSS antenna with Artificial Magnetic Conductor," 2017 Progress in Electromagnetic Research Symposium - Spring (PIERS), St. Petersburg, Russia, IEEE 2017

5. Philip R. R. Strode, Paul D. Groves, "GNSS Multipath Detection Using Three Frequency Signal to Noise Measurement, " Springer 2015

6. R. Wu, W. Wang, D. Lu, L. Wang, and Q. Jia, "Adaptive Interference Mitigation in GNSS," (Navigation: Science and Technology), 2018

7. S. Atalla, S. Tarapiah, K. Aziz, and S. H. Ismail, "Smart Real-Time Healthcare Monitoring and Tracking System using GSM/GPS Technologies," International Journal of Computer Applications, 142(14): pp. 19-26, May 2016

8. O, Yigit, Y. Asci, I. Sisman, K. Yegin, "Beam Switched Antenna Design for Jamming Mitigation of Legacy GPS Receivers, " 24th Telecommunications Forum (TELFOR), IEEE, pp. 1-3, 2016

9. G. Sateesh Kumar, G. Sasi Bhushana Rao, and M. N. V. S. S. Kumar, "GPS signal rician fading model for precise navigation in urban environment," Indian Journal of Radio and Space Physics, Vol. 42, pp. 192-196, June 2013.

10. J. Wong, T. Sanna, I. Nazrin, Ch. Kok, "Indoor Navigation and Localisation Application System," 2016 3rd International Conference on Electronic Design (ICED), IEEE, pp. 327-331, 2016

11. R. A. Poisel, "Information Warfare and Electronic Warfare Systems, " Artech House, 2013

12. K.Yegin, "On-Vehicle GPS Antenna Measurement," in IEEE Antennas and Wireless Propagations Letters, pp. 488-491, 2007

13. Jack K Holmes, "Spread Spectrum Systems for GNSS and Wireless Communications," Artech House, 2007

14. M. Slowik, D. Oldziej, Z. Gosiewski, "Integration and In-Field Gains Selection of Flight and Navigation Controller for Remotely Piloted Aircraft System," Acta Mechanica et Automatica, vol. 11, no. 1, pp. 33-37, 2017

15. R. A. Poisel, "Modern Communications Jamming Principles and Techniques, " Artech House, 2011

16. G. Sateesh Kumar, G. Sasi Bhushana Rao, and M. N. V. S. S. Kumar, "GPS Signal Short Term Propagation Characteristics Modeling in Urban Areas for Precise Navigation Applications," pp. 192-199, 2013

17. B. Oszczak, K. Olek, E. Sitnik, A. Czarnocka, "GNSS positioning algorithm with use of uncorrelated reference point indicators," 15th International Multidisciplinary Scientific Geo Conference SGEM 2015, Vol. II, pp. 277-282, 2015

18. F. Dovis., "GNSS Interference Threats and Countermeasures, (Gnss Technology and Applications) first Edition, ” Artech House, 2007

19. W. Walendziuk, D. Oldziej, D. P. Binczyk, M. Slowik," Ground control station software design for micro aerial vehicles, " Proceedings Volume 10445, Photonics Applications in Astronomy, Communications, Industry, and High Energy Physics Experiments 2017, (2017)

20. D. Zhang, F. Xia, Z. Yang, L. Yao, W. Zhao, "Localization Technologies for Indoor Human Tracking," 5th International Conference on Future Information Technology (FutureTech), IEEE Communications Surveys \& Tutorials, vol. 11, pp. $1-6,2010$

21. I. Petrovski, T. Tsujii, "Digital Satellite Navigation and Geophysics: A Practical Guide with GNSS Signal Simulator and Receiver Laboratory," Cambridge University Press, 2012

22. R. Poisel, "Introduction to Communication Electronic Warfare Systems, " Artech House, 2008

23. B. Parkinson, and J. J. Spilker, "Global Positioning System: Theory And Applications Vol. 1," American Institute of Aeronautics and Astronautics, Inc., 1996

24. I. Moir, A. Seabridge, "Design and Development of Aircraft Systems," Second Edition, John Wiley \& Sons, Ltd., 2013

25. L. Setlak, and R. Kowalik, "Mathematical modeling and simulation of selected components on-board autonomous power supply system (ASE), in accordance with the concept of a more electric aircraft (MEA)," 2017 18th International Scientific Conference on Electric Power Engineering (EPE), Czech Republic, pp. 1-6, IEEE 2017

26. K. Emandi, M. Ehsani, "Aircraft power systems: technology, state of the art, and future trends, Aerospace and Electronic Systems, " IEEE 2000

27. B. Oszczak, M. Grzegorzewski, J. Cwiklak, "Solution for Inter-Satellite Linked Space-Time Network Using Reference and Transition Point Indicators," Proceedings of the 27th International Technical Meeting of the Satellite Division of the Institute of Navigation (ION GNSS 2014), pp. 23632370, 2014

28. M. Grzegorzewski, "Results of a Research Predicting the Position of an Aircraft during Approach and Landing using the Bessel Function," Journal of Theoretical and Applied Mechanics, Vol. 51, No. 4, pp. 915-926, 2013

29. L. Setlak, R. Kowalik "Components of Variable Frequency of Power Supply System ASE of Modern Aircraft in Accordance with the Concept of a More Electric Aircraft MEA," Lecture Notes in Engineering and Computer Science: Proceedings of WCECS 2017, San Francisco, USA, pp. 243-248, 2017

30. M. Grzegorzewski, A. Ciecko, S. Oszczak, and D. Popielarczyk, Autonomous and EGNOS Positioning Accuracy Determination of Cessna Aircraft on the Edge of EGNOS Coverage, Proceedings of the 2008 National Technical Meeting of the Institute of Navigation - NTM 2008, 2008 National Technical Meeting of the Institute of Navigation, pp. 407-410, 2008 